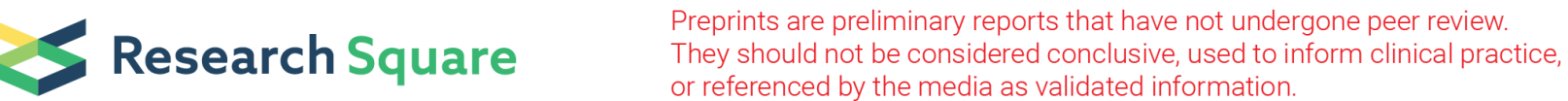

\section{Correlation Between Disability Measure and Black Hole (T1 Hypointense Lesion) Lesion Load in Brain Magnetic Resonance Imaging (MRI) of Patients With Multiple Sclerosis (MS): Protocol for a Systematic Review}

\section{Amir Valizadeh}

Tehran University of Medical Sciences https://orcid.org/0000-0001-5983-8527

\section{Mohammad Ali Sahraian}

Tehran University of Medical Sciences

\section{Mohammad Reza Fattahi}

Tehran University of Medical Sciences

Mana Moassefi ( $\square$ Moassefi@gmail.com )

Tehran University of Medical Sciences, Building no.1, Northern gate of the university https://orcid.org/0000-0002-0111-7791

\section{Protocol}

Keywords: Multiple Sclerosis, Magnetic Resonance Imaging, Multiple Sclerosis, Relapsing, Remitting, Multiple Sclerosis, Chronic Progressive

Posted Date: February 11th, 2021

DOl: https://doi.org/10.21203/rs.3.rs-194103/v1

License: (c) (1) This work is licensed under a Creative Commons Attribution 4.0 International License. Read Full License 


\section{Abstract}

Not applicable (It's in the protocol phase)

\section{Background}

\section{Description of the health condition and context}

Multiple sclerosis (MS) is a chronic, disabling disease that targets patients in work productive years. Prognostic factors play important roles in evaluating the cost of the disease on both individual patients and social economy. Since there is no exact method to predict MS progression confidently, four general MS phenotypes have been defined: clinically isolated syndrome (CIS), relapsing remitting MS (PRMS), secondary progressive (SPMS) and primary progressive (PPMS) [1, 2]. RRMS is the most common MS phenotype. It is characterized by noticeable exacerbation or initiation of neurologic symptoms. These attacks, also called relapses are followed by periods of recovery, called remissions. Some patients who are diagnosed with RRMS will gradually convert to a secondary progressive course which is a progressive deterioration of neurologic function over time. The other two types are not mentioned in this study.

\section{Description of the prognostic / predictive model(s) / factor(s)}

Magnetic resonance imaging (MRI) is a sensitive para clinical test for diagnosis and assessment of disease progression in MS and is often used to evaluate therapeutic efficacy. As the role of neurodegeneration in the pathophysiology of MS has become more prominent, the formation and evolution of chronic or persistent T1-hypointense lesions (black holes) have been used as markers of axonal loss and neuronal destruction to measure disease activity. On the one hand, T1 lesion load showed greater cross-sectional[3] and longitudinal[3, 4] correlations with Expanded Disability Status Scale (EDSS) scores for patients with RRMS or SPMS than did T2 lesion load. On the other hand, several other studies reported an absence of correlations between T1 hypointensity and EDSS in patients with SPMS[5-9] and RRMS[7, 10].

\section{Health outcomes}

Disability: While MS commonly causes disabling signs and symptoms in patients, there are different systems for evaluating patients' disability level. The Expanded Disability Status Scale (EDSS)[11] is one of them, which is extensively used in studies for the assessment of people with MS. EDSS is a technique of measuring disability in multiple sclerosis and observing alterations in the level of disability over time.

\section{Why it is important to do this review}

In this study we aim to review all the relevant studies, to evaluate the correlation between T1 hypointensities (Black holes) on brain MRI with disability level of the patients with MS. Our main goal is to clarify if this possible prognostic factor can be used to help determining the prognosis of patients. 


\section{Objectives}

To evaluate the correlation between T1 hypointensities (Black holes) lesion load (lesion mean volume) on brain MRI with disability level of patients with RRMS or SPMS.

With regard to objective 1 we established the following 'Population, Intervention, Outcome, Timing, Setting' (PICOTS) table (adapted according to the PICOTS system presented in by Debray in 2017[12]).

\begin{tabular}{|ll|}
\hline Population & Patients with RRMS or SPMS \\
\hline Comparator & Patients with RRMS or SPMS without the prognostic factor \\
\hline $\begin{array}{l}\text { Index (prognostic factor under } \\
\text { study) }\end{array}$ & $\begin{array}{l}\text { T1 hypointensities (Black holes) lesion load (lesion mean volume) } \\
\text { on brain MRI }\end{array}$ \\
\hline Outcomes & EDSS \\
\hline Timing & Measured at the same time as MRI was performed \\
\hline $\begin{array}{l}\text { Setting } \\
\text { RRMS: Relapsing-Remitting Multiple Sclerosis; SPMS: Secondary Progressive Multiple Sclerosis, } \\
\text { EDSS: Expanded Disability Status Scale }\end{array}$ \\
\hline
\end{tabular}

\section{Methods}

Design and methods used for this systematic review protocol are reported in line with Cochrane Prognosis Methods group checklist for protocols[13] and The PRISMA statement for reporting systematic reviews and meta-analyses[14].

\section{Criteria for considering studies for this review}

\section{Types of studies}

Retrospective and prospective cohort studies investigating disability status of RRMS and SPMS patients with T1 hypointensities using EDSS.

\section{Targeted population}

To study the correlation between $\mathrm{T} 1$ hypointensities and disability status, we included patients with a diagnosis of RRMS or SPMS based on the McDonald criteria[1, 15-17]. Diagnosis should have been made by a trained physician. We will only include adult patients with an age between 18 and 50 years. Patients will be included irrespective of their sex.

\section{Multiple Sclerosis diagnosis based on latest McDonald criteria}


- Two attacks or symptom flare-ups (lasting at least 24 hours with 30 days between attacks), plus two lesions.

- Two attacks, one lesion, and evidence of dissemination in space (or a different attack in a different part of the nervous system).

- One attack, two lesions, and evidence of dissemination in time (or finding a new lesion - in the same location - since the previous scan, or presence of immunoglobulin, called oligoclonal bands in the spinal fluid).

- One attack, one lesion, and evidence of dissemination in space and time.

- Worsening of symptoms or lesions and dissemination in space found in two of the following: MRI of the brain, MRI of the spine, and spinal fluid.

Relapsing-Remitting course: A multiple sclerosis course characterized by relapses with stable neurological disability between episodes.

Progressive course: A multiple sclerosis course characterized by steadily increasing objectively documented neurological disability independent of relapses. Fluctuations, periods of stability, and superimposed relapses might occur. Primary progressive multiple sclerosis (a progressive course from disease onset) and secondary progressive multiple sclerosis (a progressive course following an initial relapsing-remitting course) are distinguished.

\section{Types of prognostic factors}

Our prognostic factors would be T1 hypointense lesion load (lesion mean volume).

\section{Types of outcomes to be predicted}

Our outcome of interest is the disability level of patients, measured using the Expanded Disability Status Scale (EDSS)[11], a scale specifically built for rating neurologic impairment in multiple sclerosis.

\section{Search methods for identification of studies}

\section{Electronic searches}

We will search the Cochrane Library (CENTRAL), MEDLINE using PubMed, Embase, Science Citation Index - Expanded (Web of Science), and Conference Proceedings Citation Index - Science (Web of Science) for any relevant studies. Our search strategies for each database are presented in Appendix A.

\section{Data collection}

\section{Selection of studies}

Two review authors (AV and MM) will independently scan the title, abstract, or both, of every record retrieved in the literature searches to determine which studies to assess further. We will investigate the full 
text of all potentially relevant articles, resolving discrepancies through consensus or by recourse to a third review author (MF). We will prepare a flow diagram of the number of studies identified and excluded at each stage in accordance with the PRISMA flow diagram of study selection[18].

\section{Data extraction and management}

Two review authors (AV and MM) will extract key study characteristics, inclusion and exclusion criteria of participants, prognostic factor and outcome (EDSS, Duration of follow up), and baseline characteristics of study participants for all eligible studies. We will resolve any disagreements by discussion or, if required, by consultation with a third review author (MS). We will use parts of the checklist for critical appraisal and data extraction for systematic reviews of prediction modelling studies (CHARMS), which helps to evaluate prediction modelling studies[19].

\section{Assessment of risk of bias in included studies}

Two review authors (AV and MM) will assess the risk of bias of each included study. We will resolve any disagreements by consensus, or by consultation with a third review author (MF). Considering the fact that at the moment there is no standard tool for assessing risk of bias in overall prognosis studies, we will use a tailored version of the Quality In Prognosis Studies (QUIPS) tool for assessing risk of bias in studies[20], presented in Appendix B. Our tailored version of the tool is consisted of six risk of bias domains: study participation, study attrition, prognostic factor measurement, outcome measurement, study confounding, and statistical analysis and reporting. The study participation domain is consisted of five items: adequate description of the source population or population of interest, adequate description of the baseline study sample, adequate description of the sampling frame and recruitment, adequate description of the period and place of recruitment, and adequate description of inclusion and exclusion criteria. The study attrition domain is consisted of four items: description of attempts to collect information on participants who dropped out, reasons for loss to follow-up provided, adequate description of participants lost to follow-up, and no important differences between participants who completed the study and those who did not. The prognostic factor measurement domain is consisted of two items: provision of clear definition or description of the prognostic factor, and reporting of continuous variables or use of appropriate cut points. The outcome measurement domain is consisted of three items: provision of clear definition of the outcome, use of adequately valid and reliable method of outcome measurement, and use of same method and setting of outcome measurement in all study participants. The study confounding domain is consisted of the seven items: measurement of all important confounders, provision of clear definitions of the important confounders measured, adequately valid and reliable measurement of all important confounders, use of same method and setting of confounding measurement in all study participants, appropriate imputation methods used for missing confounders (if applicable), important potential confounders accounted for in the study design, and important potential confounders accounted for in the analysis. The statistical analysis and reporting domain is consisted of two items: sufficient presentation of data to assess the adequacy of the analytic strategy, and adequate statistical model for the design of the study. 


\section{Dealing with missing data}

In the case of missingness of data, if possible, we will try to contact the original investigators to request missing information. In case that was unsuccessful, we will only analyze the available information and will not try to impute any missing data.

\section{Assessment of heterogeneity}

We expect some heterogeneity between studies because of ethnicity and methodological diversity. We will report the range of the effects of the random-effects meta-analyses using prediction intervals. In a random-effects meta-analysis, the prediction interval reflects the whole distribution of effects across study populations, including the effect expected in a future study[21, 22].

\section{Data synthesis}

\section{Data synthesis and meta-analysis approaches}

Our primary aim for overall prognosis in patients that has T1 hypointense lesions in their brain MRI is to provide a transparent overview possible association between lesion size and disability levels. We expect the following outcome measures:

- Correlation coefficients ( $r$ )

We will use R version 4.0 for our meta-analysis. Most meta-analysts do not perform syntheses on the correlation coefficient itself because the variance depends strongly on the correlation. Rather, the correlation is converted to the Fisher's z scale and all analyses are performed using the transformed values[23]. For meta-analysis of correlation data, we first convert the correlation coefficients to Fisher's z scale. Then we will calculate the variance and standard error of the Fisher's $z$. We will preform metaanalysis on those values based on the random-effects model. Finally, we will convert back Fisher's $z$ to correlation coefficient ( $r$ ) for the sake of presentation.

\section{Subgroup analysis}

If at least 5 studies are available for each classification of MS in our study (RRMS and SPMS), we will perform a subgroup analysis for each of them.

\section{Sensitivity analysis}

We plan to perform sensitivity analyses to explore the influence of the following factors:

- Studies at high or unclear risk of bias

- Very long or large studies to establish the extent to which they dominate the results.

\section{Certainty of the evidence}


We will use an adapted version of the GRADE framework for prognostic factor research[24] to evaluate the certainty of our evidence.

\section{Conclusions And Summary Of Findings}

We will create a 'Summary of findings' table using Review Manager 5[25]. In that table, we will include a summary of our findings, the methods used to prepare any of them, prediction intervals for our findings, and the GRADE score for each outcome. We will justify all decisions to downgrade the certainty of evidence using footnotes, and we will make comments to aid the reader's understanding of this review where necessary.

\section{Abbreviations}

CIS: Clinically isolated syndrome

CPA: Cerebellopontine angle

EDSS: Expanded Disability Status Scale

MRI: Magnetic resonance imaging

MS: Multiple sclerosis

RRMS: Relapsing remitting multiple sclerosis

SPMS: Secondary progressive multiple sclerosis

\section{Declarations}

\section{Availability and data materials}

The datasets or analysed during the current study are available in the articles in MEDLINE, Embase, CENTRAL, and Web of Science databases.

\section{Competing interests}

All authors indicate no potential conflicts of interest regarding this review subject and its possible findings.

\section{Founding}

No grants or financial support are planned for this review. 


\section{Authors contribution}

AV and MM provided the protocol for the study.

AV developed and will run the search for studies.

$\mathrm{AV}$ and $\mathrm{MM}$ will obtain copies of studies.

AV, MM and MF will screen search results for potential eligible studies.

$\mathrm{AV}, \mathrm{MM}$ and $\mathrm{MF}$ will extract data from eligible studies.

AV will enter data in to RevMan and will carry out the analysis.

$\mathrm{AV}$ and $\mathrm{MM}$ will interpret the analysis results.

$M S, A V, M M$, and MF will draft the final review.

\section{Acknowledgements}

We would like to thanks all who helped us in the production of this paper.

\section{References}

1. Thompson, A.J., et al., Diagnosis of multiple sclerosis: 2017 revisions of the McDonald criteria. Lancet Neurol, 2018. 17(2): p. 162-173.

2. Lublin, F.D., et al., Defining the clinical course of multiple sclerosis: the 2013 revisions. Neurology, 2014. 83(3): p. 278-86.

3. Truyen, L., et al., Accumulation of hypointense lesions ("black holes") on T1 spin-echo MRI correlates with disease progression in multiple sclerosis. Neurology, 1996. 47(6): p. 1469-1476.

4. Paolillo, A., et al., Quantitative MRI in patients with secondary progressive MS treated with monoclonal antibody Campath 1H. Neurology, 1999. 53(4): p. 751-7.

5. Giugni, E., et al., MRI measures and their relations with clinical disability in relapsing-remitting and secondary progressive multiple sclerosis. Multiple Sclerosis Journal, 1997. 3(4): p. 221-225.

6. O'Riordan, J.I., et al., T1 hypointense lesion load in secondary progressive multiple sclerosis: a comparison of pre versus post contrast loads and of manual versus semi automated threshold techniques for lesion segmentation. Mult Scler, 1998. 4(5): p. 408-12.

7. Simon, J.H., et al., A longitudinal study of T1 hypointense lesions in relapsing MS: MSCRG trial of interferon beta-1a. Multiple Sclerosis Collaborative Research Group. Neurology, 2000. 55(2): p. 18592. 
8. Koudriavtseva, T., et al., Gadolinium enhanced MRI predicts clinical and MRI disease activity in relapsing-remitting multiple sclerosis. J Neurol Neurosurg Psychiatry, 1997. 62(3): p. 285-7.

9. Masek, M., et al., Secondary-progressive form of multiple sclerosis: MRI changes versus clinical status. Neuro Endocrinol Lett, 2008. 29(4): p. 461-6.

10. van Walderveen, M.A., et al., Correlating MRI and clinical disease activity in multiple sclerosis: relevance of hypointense lesions on short-TR/short-TE (T1-weighted) spin-echo images. Neurology, 1995. 45(9): p. 1684-90.

11. Kurtzke, J.F., Rating neurologic impairment in multiple sclerosis: an expanded disability status scale (EDSS). Neurology, 1983. 33(11): p. 1444-52.

12. Debray, T.P., et al., A guide to systematic review and meta-analysis of prediction model performance. BMJ, 2017. 356: p. i6460.

13. Moons, K.G., et al., Implementing systematic reviews of prognosis studies in Cochrane. Cochrane Database Syst Rev, 2018. 10: p. ED000129.

14. Liberati, A., et al., The PRISMA statement for reporting systematic reviews and meta-analyses of studies that evaluate health care interventions: explanation and elaboration. Journal of clinical epidemiology, 2009. 62(10): p. e1-e34.

15. Polman, C.H., et al., Diagnostic criteria for multiple sclerosis: 2010 revisions to the McDonald criteria. Ann Neurol, 2011. 69(2): p. 292-302.

16. Polman, C.H., et al., Diagnostic criteria for multiple sclerosis: 2005 revisions to the "McDonald Criteria". Annals of Neurology: Official Journal of the American Neurological Association and the Child Neurology Society, 2005. 58(6): p. 840-846.

17. McDonald, W.I., et al., Recommended diagnostic criteria for multiple sclerosis: guidelines from the International Panel on the diagnosis of multiple sclerosis. Ann Neurol, 2001. 50(1): p. 121-7.

18. Moher, D. and A. Liberati, A., Tetzlaff, J., \& Altman, DG (2009). Preferred reporting items for systematic reviews and meta-analyses: the PRISMA statement. BMJ. 339: p. b2535.

19. Moons, K.G., et al., Critical appraisal and data extraction for systematic reviews of prediction modelling studies: the CHARMS checklist. PLoS Med, 2014. 11(10): p. e1001744.

20. Hayden, J.A., P. Cote, and C. Bombardier, Evaluation of the quality of prognosis studies in systematic reviews. Ann Intern Med, 2006. 144(6): p. 427-37.

21. IntHout, J., et al., Plea for routinely presenting prediction intervals in meta-analysis. BMJ Open, 2016. 6(7): p. e010247.

22. Riley, R.D., J.P. Higgins, and J.J. Deeks, Interpretation of random effects meta-analyses. BMJ, 2011. 342: p. d549.

23. Rosenblad, A., Introduction to Meta-Analysis by Michael Borenstein, Larry V. Hedges, Julian PT Higgins, Hannah R. Rothstein. 2009, Wiley Online Library.

24. Huguet, A., et al., Judging the quality of evidence in reviews of prognostic factor research: adapting the GRADE framework. Syst Rev, 2013. 2(1): p. 71. 
25. Nordic Cochrane Centre, T.C.C., Nordic Cochrane Centre, The Cochrane Collaboration. Review Manager 5 (RevMan 5). Version 5.3. 2014.

\section{Appendices \\ Appendix A}

\section{Embase}

\#8 \#7 AND \#1

\#7 \#6 AND \#5

\#6 disability:ab,ti,kw

\#5 \#2 OR \#3 OR \#4

\#4 'black hole ${ }^{\star \prime}: a b, t i, k w$

\#3 (t1 NEAR/5 hypointens $\left.{ }^{\star}\right): a b, t i, k w$

\#2 (t1 NEAR/5 lesion*):ab,ti,kw

\#1 'multiple sclerosis'/exp

\section{Medline via PubMed}

Multiple Sclerosis[MeSH Terms] AND ((disability[tiab]) AND ("T1 hypointens*"[tiab] OR "T1 lesion*"[tiab] OR "black hole*"[tiab]))

\section{CENTRAL}

\#1 MeSH descriptor: [Multiple Sclerosis, Relapsing-Remitting] explode all trees

\#2 MeSH descriptor: [Multiple Sclerosis, Chronic Progressive] explode all trees

\#3 \#1 OR \#2

\#4 (t1 NEAR/5 lesion*):ab,ti,kw

\#5 (t1 NEAR/5 hypointens*):ab,ti,kw

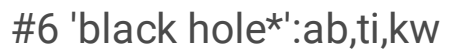


\#7 \#4 OR \#5 OR \#6

\#8 disability:ab,ti,kw

\#9 \#8 AND \#7

\#10 \#9 AND \#3

\section{Web of Science}

\#1 TS="Multiple Sclerosis"

\#2 TS=(t1 NEAR/ 5 lesion*)

\#3 TS=(t1 NEAR/ 5 hypointens*)

\#4 TS="black hole*"

\#5 \#2 OR \#3 OR \#4

\#6 TS="disability"

\#7 \#6 AND \#5

\#8 \#7 AND \#1

\section{Appendix B}




\section{Study participation: yes/no/partly/unsure/NA}

a. Adequate participation in the study by eligible individuals

b. Adequate description of the source population or population of interest

c. Adequate description of the baseline study sample

d. Adequate description of the sampling frame and recruitment

e. Adequate description of the period and place of recruitment

f. Adequate description of inclusion and exclusion criteria

Study participation: risk of bias rating (high/low/unclear)
NA: usually participants with information on T1 hypointensity in brain MRI and information on disability levels or relapse rate are selected from a greater study cohort

Source population for cohort with T1 hypointensity in MRI is clearly described

Age and number of participants at baseline is clearly described

Way of establishing the source population, selection criteria and key characteristics of the source population clearly described

Time period and place of recruitment for both baseline and follow-up visits are clearly described

Criteria used for diagnosis and classification and description of other inclusion and exclusion criteria are clearly described

High: most items are answered with 'no'; Low: all items answered with 'yes'; Unclear: most items are answered with 'unsure' Note: potentially a single item may introduce a high risk of bias, depending on study specifics

\section{Study attrition: yes/no/partly/unsure/NA}

a. Adequate response rate for study participants

b. Attempts to collect information on participants who dropped out described

c. Reasons for loss to followup provided

\section{d. Adequate description of} participants lost to follow-up

e. No important differences between participants who completed the study and those who did not

Study attrition: risk of bias rating (high/low/unclear)
NA: usually participants with information on T1 hypointensity in brain MRI and information on disability levels or relapse rate are selected from a greater study cohort

Attempts to collect information on participants who dropped out are described (e.g., telephone contact, mail, registers)

Reasons on participants who dropped out are available (e.g., deceased participants between baseline and follow-up, participants moving to another location)

Key characteristics of participants lost to follow-up are described (T1 hypointensity load)

Study authors described differences between participants completing the study and those who did not as not important or information provided to judge the differences

High: most items are answered with 'no'; Low: all items answered with 'yes'; Unclear: most items are answered with 'unsure' Note: potentially a single item may introduce a high risk of bias, depending on study specifics 
a. Clear definition or description provided

b. Adequately valid and reliable method of measurement

c. Continuous variables reported or appropriate cut points used

d. Same method and setting of measurement used in all study participants

e. Adequate proportion of the study sample had complete data

f. Appropriate methods of imputation were used for missing data

Prognostic factor measurement: risk of bias rating (high/low/ unclear)
Study authors clearly indicate how they measure T1 hypointense lesions

NA: brain MRI is the only available method for measuring T1 hypointense lesions

The exact size of the T1 hypointense lesion is reported and it is not categorized base on cut points

NA: brain MRI is the only available method for measuring T1 hypointense lesions

NA: usually participants with information on T1 hypointensity in brain MRI and information on disability levels or relapse rate are selected from a greater study cohort

NA: missing radiological size of T1 hypointense lesion can't be imputed

High: most items are answered with 'no'; Low: all items answered with 'yes'; Unclear: most items are answered with 'unsure' Note: potentially a single item may introduce a high risk of bias, depending on study specifics

\section{Outcome measurement: yes/no/partly/unsure/NA}

a. Clear definition of the outcome provided

b. Use of adequately valid and reliable method of outcome measurement

c. Use of same method and setting of outcome measurement in all study participants

Outcome measurement: risk of bias rating (high/low/unclear)
Measuring disability level using EDSS should be clearly defined

EDSS should be measured by a trained physician

Measurements of EDSS are the same for all participants
High: most items are answered with 'no'; Low: all items answered with 'yes'; Unclear: most items are answered with 'unsure' Note: potentially a single item may introduce a high risk of bias, depending on study specifics

\section{Study confounding: yes/no/partly/unsure/NA}

\section{a. Measurement of all} important confounders

b. Provision of clear definitions of the important confounders measured

c. Adequately valid and
Important confounders are: ethnicity, comedications and comorbidities, socioeconomic status

Measurement of confounders has to be clearly described 
d. Use of same method and setting of confounding measurement in all study participants

e. Appropriate imputation methods used for missing confounders (if applicable)

f. Important potential confounders were accounted for in the study design

g. Important potential confounders were accounted for in the analysis

\section{Study confounding} measurement: risk of bias rating (high/ low/unclear)
Measurements of confounders are the same for all study participants
Strategy to impute missing confounder data is described

Methods section of the publication describes strategy to account for confounders

Important confounders are accounted for in multivariable logistic regression and Cox proportional hazards models

\section{Statistical analysis and reporting: yes/no/partly/unsure/NA}

a. Sufficient presentation of data to assess the adequacy of the analytic strategy

b. Strategy for model building is appropriate and based on a conceptual framework or model

c. Statistical model is adequate for the study design

d. No selective reporting of results

Statistical analysis and reporting: risk of bias rating (high/ low/unclear)
Mean or median values, including confidence intervals or standard errors or standard deviations

NA: we do not anticipate conceptual frameworks or explicit model building strategies for this type of research question (focusing on one prognostic factor only)

Mainly correlation coefficients
NA: EDSS score and relapse rate are the only outcomes; if missing the study will be excluded

No: no or no relevant information to answer the signaling question

Partly: information is provided but not completely

Unsure: not enough information to answer signaling question with yes or no

NA (not applicable): signaling question not appropriate for this type of prognostic review 